\title{
Clinical Study \\ Study of Demarcation Line Depth in Transepithelial versus Epithelium-Off Accelerated Cross-Linking (AXL) in Keratoconus
}

\author{
Yehia Salah, Kholoud Omar, Ahmed Sherif 1 , and Sarah Azzam (iD \\ Department of Ophthalmology, Cairo University, Giza, Egypt \\ Correspondence should be addressed to Sarah Azzam; sarahazzam@ymail.com
}

Received 27 March 2019; Revised 3 July 2019; Accepted 6 September 2019; Published 26 September 2019

Academic Editor: Stefano Baiocchi

Copyright (c) 2019 Yehia Salah et al. This is an open access article distributed under the Creative Commons Attribution License, which permits unrestricted use, distribution, and reproduction in any medium, provided the original work is properly cited.

\begin{abstract}
Aim. This is a prospective interventional clinical trial to assess the depth of the demarcation line in transepithelial versus epithelium-off accelerated corneal cross-linking (AXL) in keratoconus patients. Methods. This prospective clinical trial was conducted on 40 eyes of 20 patients. Each patient had transepithelial AXL in one eye and epithelium-off AXL in the contralateral eye applying UVA light with an irradiance of $45 \mathrm{~mW} / \mathrm{cm}^{2}$ for 2.4 minutes and $30 \mathrm{~mW} / \mathrm{cm}^{2}$ for 4 minutes. The depth of the demarcation line was measured using anterior segment OCT (Topcon 3D OCT-2000) one month postoperative for both eyes. Results. The demarcation line was patchy in 50\% of the transepithelial AXL eyes, the other half showing a demarcation line at a mean depth of $183 \pm 41.6 \mu \mathrm{m}$. In the epithelium-off AXL technique, the demarcation line was well defined in all cases with a mean depth of $219 \pm 27.3 \mu \mathrm{m}$. There was a statistically significant difference in corneal demarcation line depth between transepithelial and epithelium-off techniques $(P=0.008$ and $P<0.05)$. The shallower demarcation line in the transepithelial group suggests that it is less effective. Conclusion. Based on the depth of the demarcation line, the cross-linking effect of epi-off AXL seems more efficacious than after transepithelial AXL. The future will show if the biomechanical effect will be sufficient to stop progression of keratoconus similarly after standard CXL. This trial is registered with NCT04045626.
\end{abstract}

\section{Introduction}

Keratoconus is a degenerative corneal disorder associated with corneal ectasia and thinning with eventual loss of visual acuity following myopia, irregular astigmatism, and corneal scarring [1]. Corneal collagen cross-linking is a surgical modality for increasing the corneal biomechanical strength to arrest keratoconus progression [2,3]. Standard corneal cross-linking treatment of progressive keratoconus was introduced by Wollensak et al. from Germany in 2003. It is sometimes also called the Dresden protocol and includes preoperative removal of the central corneal epithelium, application of the photosensitizer riboflavin, and UVA irradiation with an irradiance of $3 \mathrm{~mW} / \mathrm{cm}^{2}$ for 30 minutes with a total surface dose of $5.4 \mathrm{~J} / \mathrm{cm}^{2}$ [4]. So-called accelerated cross-linking (AXL) has been proposed to shorten the duration of the procedure. It is based on the Bunsen-Roscoe law of reciprocity increasing the UVA irradiance and significantly reducing the irradiation time resulting in a similar overall UVA dose [5]. Transepithelial cross-linking, gained ground by leaving epithelium intact, improving patient comfort and minimizing the risk of infection associated with epithelial removal. However, its efficacy remained controversial as the epithelial barrier limits the depth and amount of cross-linking, as evidenced by a shallow and uneven distribution of the keratocytes apoptosis on confocal microscopy and ectatic progression in many clinical trials as compared to epithelium-off CXL [6]. The stratified corneal epithelium with tight junctions presents as a lipophilic barrier to the absorption of the hydrophilic riboflavin macromolecule. Standard riboflavin formulations were modified with addition of EDTA, benzalkonium chloride (BAC), and trometamol, which loosen up the epithelial junctions, enhancing the permeability and riboflavin diffusion across the epithelium and superficial stroma [7].

Studies have shown that the effective depth of standard CXL is confined to the anterior $300 \mathrm{um}$ of the cornea as demonstrated by the depth of the keratocyte apoptosis with lacunar edema on histology and the clinical demarcation line [5]. 
The purpose of our study is to evaluate and compare the depth of the corneal stromal demarcation line using ASOCT after transepithelial cross-linking versus accelerated epi-off cross-linking procedures.

\section{Patients and Methods}

This prospective comparative interventional study comprised patients with progressive keratoconus. The study was approved by the local ethical committee and followed the principles of the Declaration of Helsinki. 40 eyes of 20 patients were divided into 2 groups: Group A: transepithelial AXL; Group B: epithelium-off AXL. Inclusion criteria were progressive keratoconus, grade 1-2 keratoconus (AmslerKrumeich classification) Snellen best corrected visual acuity of 0.4 or better, and pachymetry of $400 \mu \mathrm{m}$ or more at thinnest location.

Progressive keratoconus was defined as an increase in $k_{\max }$ of 1.00 diopter [D], an increase of manifest refraction cylinder of $1.0 \mathrm{D}$, or an increase of manifest refraction spherical equivalent of $0.5 \mathrm{D}$ over the period of 1 year. CXL treatment was performed with a KXL I UVA source (Avedro Inc., Waltham, MS, USA).

In transepithelial AXL, ParaCel ${ }^{\mathrm{TM}}$ (containing 0.25\% riboflavin and benzalkonium chloride) was instilled in the form of 1 drop every 1.5 minutes for 4.5 minutes, and then VibeX Xtra was instilled in the form of 4 drops at 5.5 minutes followed by 1 drop at 6.5 minutes and again every 1.5 minutes for three times. Total soak time of riboflavin was 11 minutes followed by UVA irradiation with an intended irradiance of $45 \mathrm{~mW} / \mathrm{cm}^{2}$ (total surface dose of $7.2 \mathrm{~J} / \mathrm{cm}^{2}$ ) for 2.4 minutes.

In epithelium-off AXL, the corneal epithelium was removed using a blunt spatula (8.0 to $9.0 \mathrm{~mm}$ in diameter). VibeX Rapid (riboflavin 0.1\%) was instilled on the center of the cornea every 2 minutes for 10 minutes followed by ultraviolet-A irradiation with an intended irradiance of $30 \mathrm{~mW} / \mathrm{cm}^{2}$ (total surface dose of $7.2 \mathrm{~J} / \mathrm{cm}^{2}$ ) for 4 minutes. At the end of the procedure, a bandage contact lens was placed over the cornea and removed after full epithelization (postoperative 4-5 days). Postoperative medications included moxifloxacin 0.5\% (Vigamox, Alcon, Inc., Canada) 4 times daily for 2 weeks and artificial tears 4 times daily for one month with ascorbic acid for one month. After removal of contact lens, patients received fluoromethelone acetate 4 times daily tapered over 2 weeks.

2.1. Evaluation of Depth of the Demarcation Line. Anterior segment optical coherence tomography (AS-OCT) (Topcon 3D OCT-2000) was performed to evaluate the corneal stroma for the presence of the demarcation line 1 month postoperative by an independent observer unaware of the purpose of this study. The demarcation line measurements of this study include the epithelial thickness of about $50 \mu \mathrm{m}$.

\section{Statistical Analysis}

Microsoft EXCEL 2010 and SPSS of Windows software (version 20.0, SPSS Inc.) were used for statistical analysis.
Normality of all data samples was first checked by means of the Kolmogorov-Smirnov test. Then $t$-test was possible, and the Student $t$-test for paired data was performed for all parameter comparisons between preoperative and postoperative examinations using the same level of significance $(P<0.05)$ in all cases. And in comparing the 2 groups we used the unpaired $t$-test.

\section{Results}

This clinical prospective study included 40 eyes of 20 patients; each patient had transepithelial CXL in one eye and epithelium-off CXL in the other. 11 females and 9 males were included in the study. The mean patient age was 32 (ranging from 16 to 40 years). The mean preoperative CCT was $484.55 \pm 35.47 \mu$ in the transepithelial group and $477.75 \pm$ $37.06 \mu$ in the epi-off group $(P=0.557)$.

4.1. Demarcation Line One Month Postoperatively. The demarcation line was patchy and ill-defined in 50\% of the transepithelial eyes, the depth of which could not be assessed (Figure 1). The other half showed a clear demarcation line. However, there was a clear demarcation line in all epithelium-off eyes. There was a statistically significant difference in the incidence of a patchy corneal demarcation line between transepithelial and epithelium-off techniques $(P=0.001$ and $P<0.05)$.

\subsection{Demarcation Line Depth One Month Postoperatively.} The mean depth of the corneal demarcation line was $183 \mu \mathrm{m}$ in the transepithelial technique and $219 \mu \mathrm{m}$ in the epithelium-off technique (Figures 2 and 3 ). There was a statistically significant difference in corneal demarcation line depth between transepithelial and epithelium-off techniques $(P=0.008$ and $P<0.05$; Table 1$)$.

4.3. Postoperative Complications. Grade I corneal haze (graded according to Fantes scoring) was found in 4 eyes only in transepithelial CXL and in 9 eyes in epithelium-off CXL. No delayed epithelialization or infective keratitis was seen in either group.

\section{Discussion}

Corneal CXL with riboflavin and ultraviolet-A (UVA) is a technique to strengthen corneal tissue [8]. The corneal epithelium is the critical obstacle to riboflavin permeation into the corneal stroma $[8,9]$. However, the removal of epithelium can cause some complications such as severe postoperative pain, temporary visual blurring, epithelial healing problems, haze, viral reactivation, and even corneal melting. To prevent these serious problems, some researchers have performed a number of studies on a special riboflavin solution, which can penetrate the intact epithelium, such as benzalkonium chloride, EDTA, and trometamol [7].

In the epi-off group, our results were comparable to Moramarco et al. [10] and Jiang et al. [11] who performed 


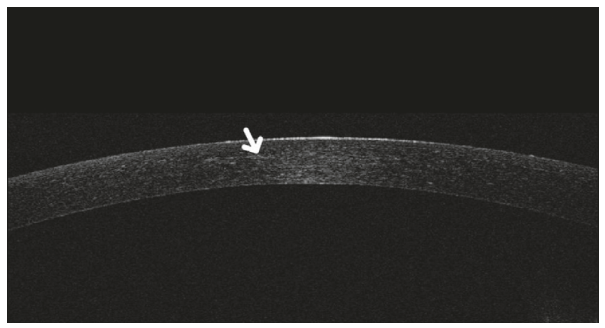

Figure 1: A patchy corneal demarcation line (white arrow) after transepithelial cross-linking.

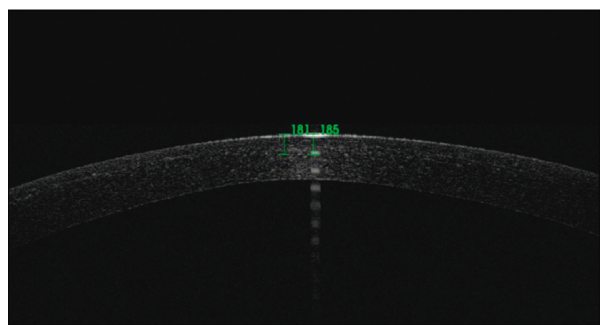

Figure 2: A barely visible corneal demarcation line at a central depth of $185 \mu \mathrm{m}$ after transepithelial cross-linking.

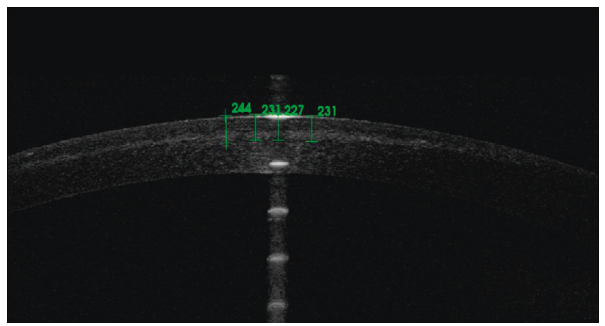

Figure 3: A corneal demarcation line at a central depth of $227 \mu \mathrm{m}$ after epithelium-off cross-linking.

TABLE 1: Summary of results.

\begin{tabular}{|c|c|c|c|c|c|}
\hline & Transepithelial, mean & SD & Accelerated epithelium-off, mean & SD & $P$ value \\
\hline Central corneal thickness & 484.55 & 35.47 & 477.75 & 37.06 & 0.557 \\
\hline Demarcation line depth & 183.0 & 41.60 & 219.30 & 27.30 & 0.008 \\
\hline
\end{tabular}

epi-off AXL using pulsed UVA light with 8 minutes of UVA exposure at $30 \mathrm{~mW} / \mathrm{cm}^{2}$ and energy dose of $7.2 \mathrm{~J} / \mathrm{cm}^{2}$. The mean depth of the stromal demarcation line was $213 \pm 47.38 \mu \mathrm{m}$ and $201.64 \pm 27.72 \mu \mathrm{m}$, respectively.

Bouheraoua et al. showed similar results using irradiance of $30 \mathrm{~mW} / \mathrm{cm}^{2}$ for 3 minutes $\left(5.4 \mathrm{~J} / \mathrm{cm}^{2}\right.$ surface dose). The corneal demarcation line was visible in $87.5 \%$ (mean depth $184.2 \pm 38.9 \mu \mathrm{m})$ in the A-CXL group [12].

The depth of the demarcation line in our study of epithelium-off cases was found to be shallower (mean $219 \mu$ ) than in other studies using different accelerated protocols. In a study by Kymionis et al., patients were treated for 10 minutes in an intended irradiance of $9.0 \mathrm{~mW} / \mathrm{cm}^{2}$ corresponding to a total surface dose of $5.4 \mathrm{~J} / \mathrm{cm}^{2}$. The mean depth of the corneal stromal demarcation line was $288.46 \pm 42.37 \mathrm{~mm}$ [13]. Another study by Kymionis et al., using high intensity CXL protocol of $18 \mathrm{~mW} / \mathrm{cm}^{2} \mathrm{UVA}$ irradiation for 7 minutes, the mean corneal stromal demarcation line depth was $313.37 \pm 48.85$ um [14].
Similarly, Lhuillier et al. using an accelerated protocol of $10 \mathrm{~min}, 9 \mathrm{~mW} / \mathrm{cm}^{2}$ ultraviolet-A, corresponding to a total surface dose of $5.4 \mathrm{~J} / \mathrm{cm}^{2}$ reported a mean depth of the stromal demarcation line of $331.2 \pm 62.7 \mu \mathrm{m}$ [15].

Our results on AXL were markedly different from Yam et al. who used a classic protocol of $3.0 \mathrm{~mW} / \mathrm{cm}^{2}$ for 30 minutes (surface dose $5.4 \mathrm{~J} / \mathrm{cm}^{2}$ ). The median CXL demarcation line depth at the central cornea was $323.0 \pm 48.6$, suggesting a significantly weaker cross-linking effect of AXL compared to standard CXL [5].

As regards for the transepithelial AXL technique, UVA irradiation was performed for 2.4 minutes, with an intended power of $45 \mathrm{~mW} / \mathrm{cm}^{2}$, giving a total energy of $7.2 \mathrm{~J} / \mathrm{cm}^{2} .50 \%$ of the cases had a patchy demarcation line and $50 \%$ had a demarcation line at a mean depth of $183 \mu \mathrm{m}$.

These results were similar to Artola et al. who reported a demarcation line depth between 153 and $230 \mu \mathrm{m}$, with a mean value of $205.19 \mu \mathrm{m}$ [16]. 
Our study's findings support the review by Liu et al. who collected eligible studies retrieved from four electronic databases, including CENTRAL, ClinicalTrials.gov, PupMed, and Ovid MEDLINE. 24 comparative studies on transepithelial cross-linking compared with standard crosslinking were included and pooled for analysis. The demarcation line of standard CXL was significantly deeper than that of transepithelial cross-linking; the mean difference of the depth of the demarcation line (DDL) was $133.49 \mu \mathrm{m}$ [17].

Both transepithelial and epi-off AXL have been shown to be biomechanically less efficacious than standard CXL [5]. The weaker effect is also reflected by the present study with the difference in the DL depth and the often patchy and irregular configuration or even absence of the DL in transepithelial AXL probably due to an inhomogenous distribution of the photosensitizer riboflavin [18].

In conclusion, we found that the demarcation line was not consistent in transepithelial cases, and it was found to be more superficial than in standard CXL as has also been found by other studies with different AXL protocols. A shallower demarcation line demonstrates a weaker crosslinking effect. It has been shown that the CXL effect has a natural saturation effect which is maximally high in a specific tissue layer. The effect can only be increased further by increasing the volume of cross-linked tissue which is reflected and revealed by the greater depth of DL [5].

The future will show if the clearly weaker cross-linking effect of epi-off and transepithelial AXL compared to standard CXL will be sufficient to stop progression of keratectasia.

\section{Data Availability}

The data used to support the findings of this study are available from the corresponding author upon request.

\section{Conflicts of Interest}

The authors declare that they have no conflicts of interest.

\section{References}

[1] Y. S. Rabinowitz, "Keratoconus," Survey of Ophthalmology, vol. 42, no. 4, pp. 297-319, 1998.

[2] G. Wollensak, "Crosslinking treatment of progressive keratoconus: new hope," Current Opinion in Ophthalmology, vol. 17, no. 4, pp. 356-360, 2006.

[3] E. B. Ozgurhan, B. I. Sezgin Akcay, Y. Yildirim, G. Karatas, T. Kurt, and A. Demirok, "Evaluation of corneal stromal demarcation line after two different protocols of accelerated corneal collagen cross-linking procedures using anterior segment optical coherence tomography and confocal microscopy," Journal of Ophthalmology, vol. 2014, Article ID 981893, 5 pages, 2014.

[4] G. Wollensak, E. Spoerl, and T. Seiler, "Riboflavin/ultravioletA-induced collagen crosslinking for the treatment of keratoconus," American Journal of Ophthalmology, vol. 135, no. 5, pp. 620-627, 2003.

[5] C. Mazzotta, G. Wollensak, F. Raiskup, A. M. Pandolfu, and E. Spoerl, "The meaning of the demarcation line after
riboflavin-UVA corneal collagen crosslinking," Expert Review of Ophthalmology, vol. 14, no. 2, pp. 115-131, 2019.

[6] A. Caporossi, C. Mazzotta, A. L. Paradiso, S. Baiocchi, D. Marigliani, and T. Caporossi, "Transepithelial corneal collagen crosslinking for progressive keratoconus: 24-month clinical results," Journal of Cataract \& Refractive Surgery, vol. 39, pp. 1157-1163, 2013.

[7] C. Koppen, K. Wouters, D. Mathysen, J. Rozema, and M. J. Tassignon, "Refractive and topographic results of benzalkonium chloride-assisted transepithelial cross linking," Journal of Cataract \& Refractive Surgery, vol. 38, no. 6, pp. 1000-1005, 2012.

[8] E. Spoerl, M. Huhle, and T. Seiler, "Induction of cross-links in corneal tissue," Experimental Eye Research, vol. 66, no. 1, pp. 97-103, 1998.

[9] E. Spoerl, M. Mrochen, D. Sliney, S. Trokel, and T. Seiler, "Safety of UVA-riboflavin cross-linking of the cornea," Cornea, vol. 26, no. 4, pp. 385-389, 2007.

[10] A. Moramarco, A. Iovieno, A. Sartori, and L. Fontana, "Corneal stromal demarcation line after accelerated crosslinking using continuous and pulsed light," Journal of Cataract \& Refractive Surgery, vol. 41, no. 11, pp. 2546-2551, 2015.

[11] L. Z. Jiang, W. Jiang, and S. Y. Qiu, "Conventional vs. pulsedlight accelerated corneal collagen cross-linking for the treatment of progressive keratoconus: 12-month results from a prospective study," Experimental and Therapeutic Medicine, vol. 14, no. 5, pp. 4238-4244, 2017.

[12] N. Bouheraoua, L. Jouve, M. El Sanharawi et al., "Optical coherence tomography and confocal microscopy following three different protocols of corneal collagen-crosslinking in keratoconus," Investigative Opthalmology \& Visual Science, vol. 55, no. 11, pp. 7601-7609, 2014.

[13] G. D. Kymionis, K. I. Tsoulnaras, M. A. Grentzelos et al., "Corneal stroma demarcation line after standard and highintensity collagen crosslinking determined with anterior segment optical coherence tomography," Journal of Cataract \& Refractive Surgery, vol. 40, no. 5, pp. 736-740, 2014.

[14] G. D. Kymionis, K. I. Tsoulnaras, D. A. Liakopoulos, C. A. Skatharoudi, M. A. Grentzelos, and N. G. Tsakalis, "Corneal stromal demarcation line depth following standard and a modified high intensity corneal cross-linking protocol," Journal of Refractive Surgery, vol. 32, no. 4, pp. 218-222, 2016.

[15] L. Lhuillier, C. Ghetemme, M. Boiché et al., "Visibility and depth of the stromal demarcation line after corneal collagen cross-linking using anterior segment optical coherence tomography: comparison between isoosmolar and hypoosmolar riboflavin," Cornea, vol. 37, no. 5, pp. 567-573, 2018.

[16] A. Artola, D. P. Piñero, P. Ruiz-Fortes, R. Soto-Negro, and R. J. Pérez-Cambrodí, "Clinical outcomes at one year following keratoconus treatment with accelerated transepithelial cross-linking," International Journal of Ophthalmology, vol. 10, no. 4, pp. 652-655, 2017.

[17] Y. Liu, Y. Liu, Y. N. Zhang et al., "Systematic review and metaanalysis comparing modified cross-linking and standard cross-linking for progressive keratoconus," International Journal of Ophthalmology, vol. 10, no. 9, pp. 1419-1429, 2017.

[18] G. Wollensak and E. Iomdina, "Biomechanical and histological changes after corneal crosslinking with and without epithelial debridement," Journal of Cataract \& Refractive Surgery, vol. 35, no. 3, pp. 540-546, 2009. 


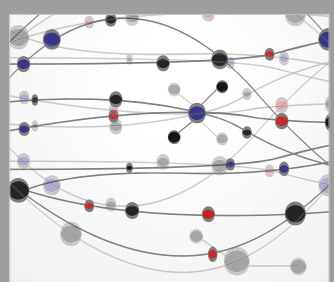

The Scientific World Journal
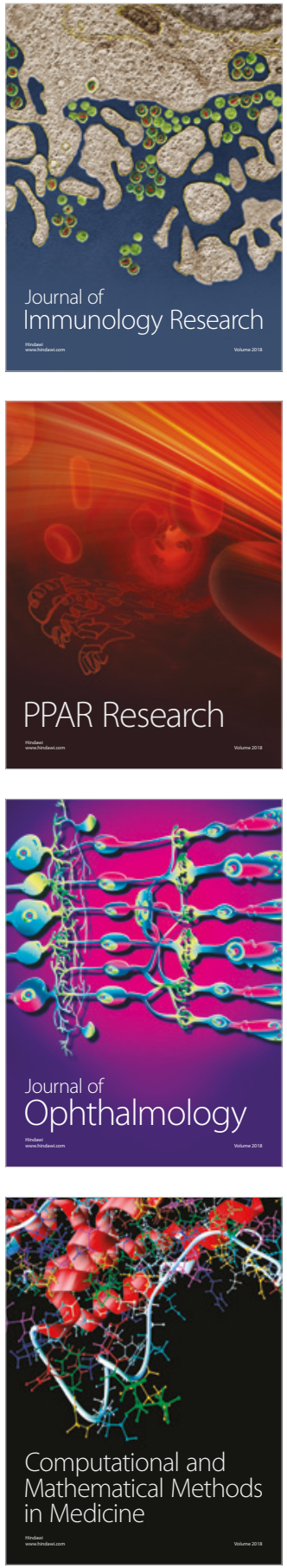

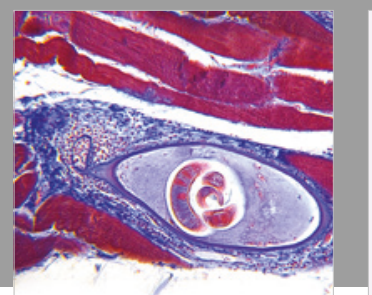

Gastroenterology Research and Practice

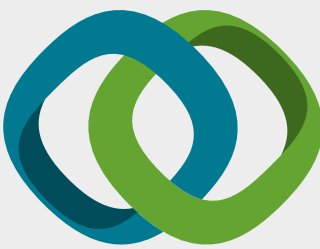

\section{Hindawi}

Submit your manuscripts at

www.hindawi.com
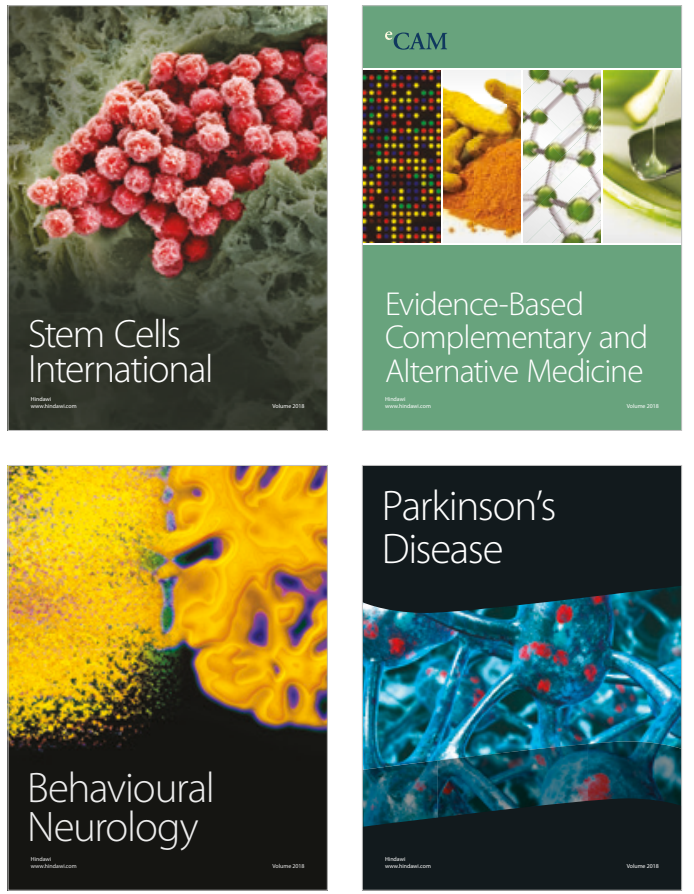

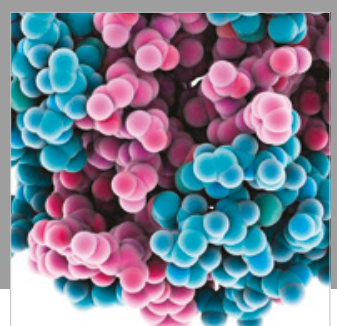

ournal of

Diabetes Research

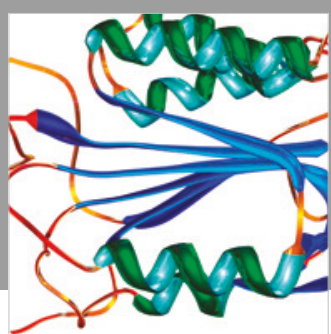

Disease Markers
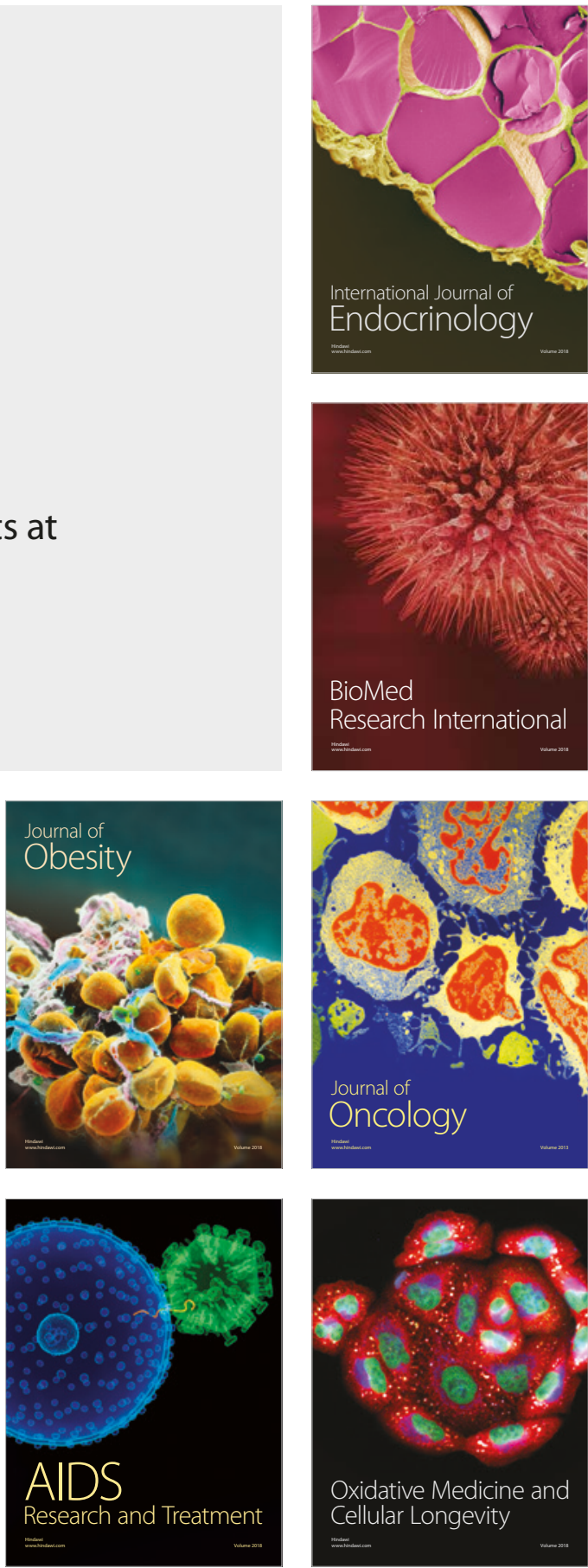